\title{
Possible role of fetuin-B in the preterm delivery in the rat
}

\author{
E. Ducza, AB. Seres, R. Gáspár
}

University of Szeged, Department of Pharmacodynamics and Biopharmacy, Szeged, Hungary

\section{INTRODUCTION}

Fetuin-B is an inhibitor of basic calcium phosphate, preventing unwanted calcification. The low level of fetuin could be associated with an increased risk of atherosclerosis and ectopic microcalcifications in soft tissues and the rupture of the membranes leading to preterm delivery. Our aims were (1) to examine the alterations of mRNA expression of the fetuin-B in the late pregnant rat uterus, furthermore (2) to investigate the fetuin-B expression in hormonally- and LPS- (lipopolysaccharide) induced preterm labour. (3) Moreover we examined the effect of farnesoid $X$ receptor $(F X R)$ agonist ursodeoxycholic acid (UDCA) on fetuin-B expression.

\begin{abstract}
METHODS
RT-PCR studies: Changes in Fetuin-B mRNA expression were measured by real-time PCR in the rat uterus.

Hormonally-induced preterm delivery: Delivery was induced before term by subcutaneous injection of antigestagen mifepristone at 9 AM in 19-daypregnant rats. Mifepristone treatment was followed by intravaginal application of prostaglandin E2 at 4 PM. Control rats received vehicle injection and gel application only

LPS-induced preterm delivery: The rats will be treated with bacterial lipopolysaccharide (LPS). Escherichia coli endotoxin (i.p. $125 \mathrm{mg} /$ day) for three consecutive days from day 18 of pregnancy, in the early morning hours, to evoke preterm birth, which occurred in the early afternoon of day 20.

UDCA treatment: From pregnancy day 18 the animals were treated with UDCA (100 mg/kg/day).
\end{abstract}

\section{RESULTS}

(1) The fetuin-B mRNA expression was the highest on days 20 and significantly decreased by last day of pregnancy (day 22) (Fig. 1).

(2) In hormonally-induced preterm labour the changes of fetuin-B mRNA expression was similarly to the last day of normal pregnancy. On the other hand, the fetuin-B mRNA level significantly increased in the LPS-induced preterm birth compared to the last day of normal pregnancy and hormonally-induced preterm birth. (Fig. 2)

(3) The pre-treatment of UDCA markedly increased the fetuin-B mRNA level in the uterus of day of term. In hormonally induced preterm we did not find any significant changes in mRNA level by UDCA, but significant decreased of fetuin-B mRNA expression in LPS model.(Fig. 3)

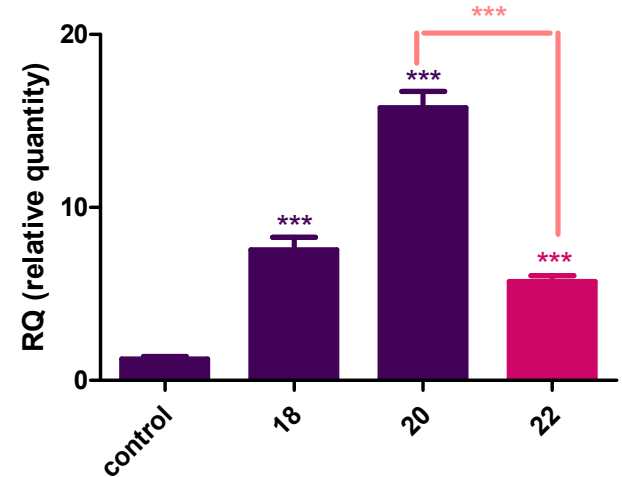

days of pregnancy

Figure 1. The changes in mRNA expression of the fetuin-B in the different days $(\mathbf{1 8}, \mathbf{2 0}$ and $\mathbf{2 2})$ of pregnant rat uterus. control: non-pregnant uterus.

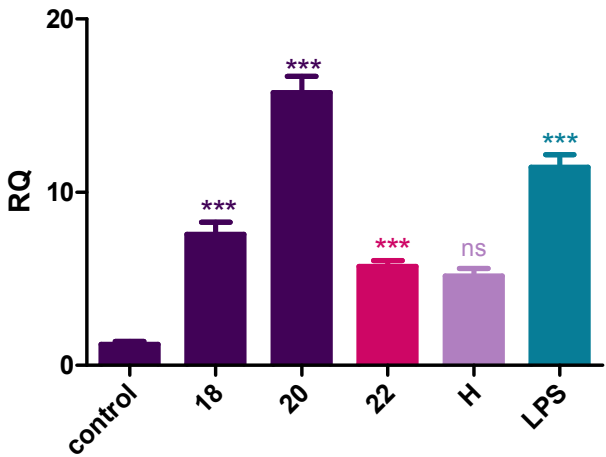

days of pregnancy

Figure 2. The changes in mRNA expression of the fetuin-B in the different days (18, 20 and 22) of pregnant rat uterus, hormonally (H) and LPS-induced (LPS) preterm labour control: non- pregnant uterus.
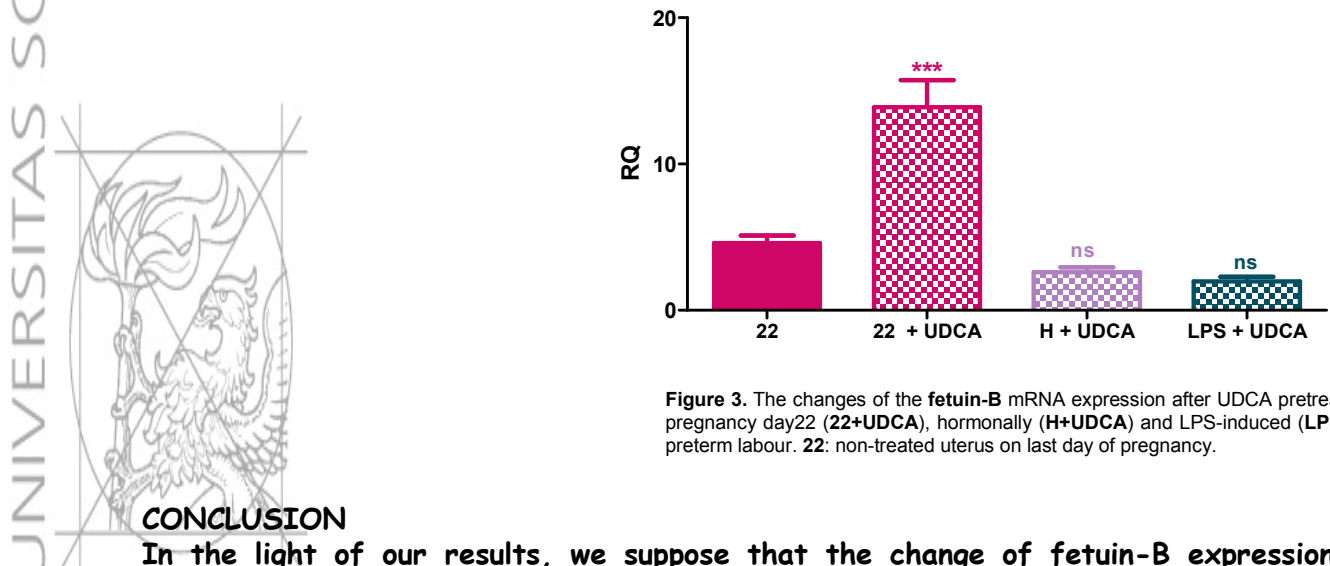

Figure 3. The changes of the fetuin-B mRNA expression after UDCA pretreatment on pregnancy day22 (22+UDCA), hormonally (H+UDCA) and LPS-induced (LPS+UDCA) preterm labour. 22: non-treated uterus on last day of pregnancy.

In the light of our results, we suppose that the change of fetuin-B expression has a possible role in the initiation of delivery in the rat: furthermore the detection of this protein can be diagnostic value for reason of the preterm birth. Further studies are required to clarify the putative role of FXR agonists (UDCA) in the control of delivery or preterm birth. 